\title{
Secondary analysis of electronically monitored medication adherence data for a cohort of hypertensive African-Americans
}

This article was published in the following Dove Press journal:

Patient Preference and Adherence

21 March 2012

Number of times this article has been viewed

\author{
George J Knafl' \\ Antoinette Schoenthaler ${ }^{2}$ \\ Gbenga Ogedegbe ${ }^{2}$ \\ 'School of Nursing, University of \\ North Carolina at Chapel Hill, \\ Chapel Hill, NC, USA; ${ }^{2}$ Center for \\ Healthful Behavior Change, New York \\ University School of Medicine, \\ New York, NY, USA
}

Background: Electronic monitoring devices (EMDs) are regarded as the "gold standard" for assessing medication adherence in research. Although EMD data provide rich longitudinal information, they are typically not used to their maximum potential. Instead, EMD data are usually combined into summary measures, which lack sufficient detail for describing complex medication-taking patterns. This paper uses recently developed methods for analyzing EMD data that capitalize more fully on their richness.

Methods: Recently developed adaptive statistical modeling methods were used to analyze EMD data collected with medication event monitoring system (MEMS ${ }^{\mathrm{TM}}$ ) caps in a clinical trial testing the effects of motivational interviewing on adherence to antihypertensive medications in a cohort of hypertensive African-Americans followed for 12 months in primary care practices. This was a secondary analysis of EMD data for 141 of the 190 patients from this study for whom MEMS data were available.

Results: Nonlinear adherence patterns for 141 patients were generated, clustered into seven adherence types, categorized into acceptable (for example, high or improving) versus unacceptable (for example, low or deteriorating) adherence, and related to adherence self-efficacy and blood pressure. Mean adherence self-efficacy was higher across all time points for patients with acceptable adherence in the intervention group than for other patients. By 12 months, there was a greater drop in mean post-baseline blood pressure for patients in the intervention group, with higher baseline blood pressure values than those in the usual care group.

Conclusion: Adaptive statistical modeling methods can provide novel insights into patients' medication-taking behavior, which can inform development of innovative approaches for tailored interventions to improve medication adherence.

Keywords: adaptive statistical modeling, hypertension, medication adherence, Medication Event Monitoring System

\section{Introduction}

An increasingly common approach for measuring medication adherence is the electronic monitoring device (EMD), often the Medication Event Monitoring System (MEMSTM) cap (AARDEX Group Ltd, Sion, Switzerland). EMDs have been used to monitor adherence with antihypertensive medications ${ }^{1-7}$ and with a variety of other medications. They provide rich information on the timing of events, but most analyses of EMD data focus on simple summary adherence measures, such as percent prescribed doses taken and percent prescribed doses taken at the correct time interval. ${ }^{8}$ These summary adherence measures do not use EMD data to their maximum potential, in part because they are based on an implicit assumption of constant adherence over time,
Correspondence: George J Knaf School of Nursing, University of North Carolina at Chapel Hill, Campus Box 7460, Chapel Hill, NC, 27599-7460, USA

Tel + I 9198439686

Fax + I 9198439969

Email gknafl@unc.edu 
which often does not hold. In addition, summary adherence measures do not provide sufficient detail to describe the complex patterns of medication-taking behavior. In contrast, Düsing et al and Vrijens et al modeled daily adherence with logistic regression methods adjusted for correlation using generalized estimating equations. ${ }^{2,7}$

The adaptive statistical modeling (ASM) approach is intermediate between the summary measure approach and modeling of daily adherence. ${ }^{9}$ ASM analyses are based on counts and rates of EMD events (for example, MEMS cap openings) within distinct time periods during a patient's participation in a study. These counts/rates are modeled using Poisson regression models based on multiple power transforms of time with arbitrary real-valued powers. The transforms and their powers are generated through an adaptive (ie, adapted to the data) process based on a heuristic, rule-based search, and these models provide curves representing individual patient adherence patterns that are clustered into adherence types. Aloia et al used a similar approach, analyzing EMD adherence to continuous positive airway pressure treatment for patients with obstructive sleep apnea using time series models and classification of patient adherence based on parameter estimates for those models..$^{10}$ ASM methods have been used to provide a variety of insights into adherence using EMD data, for example, establishing the efficacy of interventions, identifying signature adherence types, and matching patients to interventions. ${ }^{11}$ However, ASM methods have currently only been applied to EMD adherence data for antiretroviral medications..$^{12,13}$

This paper reports on the use of ASM methods to analyze EMD data on adherence to antihypertensive medications in a cohort of African-American patients with hypertension. Findings from reported analyses provide novel insights into adherence for these patients. These findings are of importance with regard to medication adherence in the particular case of hypertensive African-American patients, and suggest the need for similar analyses in general electronically monitored adherence settings.

\section{Materials and methods \\ Motivational interviewing study}

The motivational interviewing (MINT) study was a randomized, controlled trial designed to compare the effects of MINT and usual care on medication adherence in hypertensive African-Americans followed in two primary care practices in New York City. ${ }^{4,14}$ The study methods have been reported elsewhere. ${ }^{4}$ Briefly, patients were approached to participate in the study during regular clinic visits. All patients were provided with written informed consent forms approved by the institutional review board of Weill Cornell Medical College and Columbia University Medical Center. After the patients consented to participate in the study, research assistants conducted baseline interviews, gave the patients a pill bottle with a MEMS cap to record openings, and instructed them on how to use it. When patients were prescribed multiple antihypertensive medications, their providers were asked to choose a medication taken once daily to be placed in the bottle. Following the baseline assessment, patients were randomly assigned to either the MINT or usual care group. Follow-up assessments were carried out at 3-month intervals for a period of one year (a total of four post-baseline visits), during which patient medication adherence data were downloaded from their MEMS caps.

\section{Outcomes of MINT study}

The primary outcome was the percent prescribed doses taken, as assessed by MEMS caps. Poor adherence was defined as taking less than $80 \%$ of the prescribed doses. Secondary outcomes included within-patient changes in adherence self-efficacy, systolic blood pressure, and diastolic blood pressure from baseline to 12 months. Patient electronic medical records were reviewed for systolic and diastolic blood pressure readings at each study visit. The within-patient change in systolic/diastolic blood pressure was computed as the difference in systolic/diastolic blood pressure between the baseline and 12-month readings. Adherence self-efficacy was measured using a 25 -item Medication Adherence SelfEfficacy Scale developed to assess patients' confidence in taking their antihypertensive medications under a variety of situations that might pose difficulties for them. ${ }^{15}$ This scale used a four-point Likert-type response format from 1 (not at all sure) to 4 (extremely sure). Scores for each item were summed and averaged so that the range of possible scores was 1 to 4 , with higher scores reflecting greater self-efficacy. Cronbach's alphas ranged from 0.88 to 0.91 over 0 to 12 months. Within-patient changes in adherence self-efficacy from baseline to 12 months were computed as differences in adherence self-efficacy scores between these two time points. Adherence self-efficacy, and systolic and diastolic blood pressure data were collected at baseline and at months 3, 6, 9, and 12. Using an intent-to-treat analysis, it was found that the MINT group had better medication adherence than the usual care group. ${ }^{14}$

This paper reports on secondary analyses of EMD data and longitudinal outcomes from the MINT study using ASM methods. The analyses serve as examples of 
the application of ASM methods and their usefulness for modeling medication adherence and assessing their impact on patient outcomes.

\section{Description of ASM methods}

ASM methods were originally formulated for Poisson regression modeling of mean adherence. ${ }^{9}$ They were then extended to identify adherence types using adaptive clustering, ${ }^{12}$ to model repeated-measures data adaptively, accounting for within-patient correlation, ${ }^{16}$ and to model adherence variability adaptively, along with mean adherence over time. ${ }^{13}$ An overview of these ASM methods used is provided below. Reported ASM analyses were conducted in SAS software (version 9.2; SAS Institute Inc, Cary, NC) using SAS macros available from the corresponding author.

In ASM, each patient's EMD data are grouped into distinct, nonoverlapping time intervals. One hundred equalsized intervals are used as long as the length of these intervals is at least 3 days. Otherwise, the number of intervals is reduced so that the interval length is at least 3 days. Counts of numbers of EMD events are computed for each of the intervals, as well as associated EMD rates per unit time. The counts are modeled using Poisson regression with the canonical log link function and the appropriate offset variable to convert from a model for the counts to a model for associated rates. Models are generated for mean adherence in terms of expected values for rates as nonlinear functions of time using one or more power transforms of time with real-valued powers; thus, these are called fractional polynomial models. ${ }^{17}$

For standard Poisson regression models, variances equal the means. An extended quasi-likelihood approach ${ }^{18}$ is used with ASM methods to include dispersions that generalize the variances of linear regression models, thereby providing a measure of adherence variability. Dispersions are also modeled with fractional polynomials as nonlinear functions of time based on power transforms of time, not necessarily the same as those used for modeling mean adherence.

To visualize an individual patient's adherence pattern, observed adherence rates are plotted along with estimates of the mean adherence curve and estimates of unit error bands (ie, \pm 1 standard deviation) around that curve (see Figure 2 for examples). A measure is also computed of percent consistency of observed adherence rates with the prescribed adherence rate. This is computed as the percent of change from the prescribed rate corresponding to the associated constant unit error bands, assuming mean adherence at the prescribed rate and constant dispersion.
Power transforms for these models are adaptively selected using rule-based search techniques (for details, see Knafl et $\mathrm{al}^{13}$ ). A two-phase search process is used. First, the model is systematically expanded from the constant model by adding power transforms of primary predictors (for example, time for EMD data analyses). Next, the expanded model is contracted to a parsimonious model by removing extraneous transforms, if any, and adjusting the remaining power transforms with each removal. Models are evaluated with likelihood crossvalidation scores. Larger likelihood cross-validation scores indicate better models more consistent with the data.

The steps in the process are controlled by rules based on tolerance parameters indicating how much a penalty in reduced likelihood cross-validation scores can be tolerated at each phase to continue processing. For example, the expansion (or contraction) phase stops when the percent decrease in likelihood cross-validation scores for the next model to add (or remove) exceeds the setting of the associated tolerance parameter. Likelihood cross-validation ratio tests based on the $\chi^{2}$ distribution, analogous to likelihood ratio tests, ${ }^{19,20}$ are used to determine tolerance parameter settings that control the search. Likelihood cross-validation ratio tests can also be used to assess whether likelihood cross-validation scores for two models differ substantially (for an example, see Knafl et $\mathrm{al}^{13}$ ), but these kinds of tests are not reported in this paper.

This search process can be used to generate not only adaptive Poisson regression models for count/rate outcomes, but also adaptive regression models for continuous outcomes and adaptive logistic regression models for dichotomous outcomes. The process can also be used to generate adaptive repeated-measures models for longitudinal patient outcomes. $^{16}$

Individual-patient adherence patterns for estimated mean adherence and adherence variability at proportionally spaced times during patients' study participation are clustered into adherence types (for example high, low, deteriorating, improving). Means for available longitudinal outcomes are adaptively modeled in terms of time, treatment group, adherence type, and associated interactions, while accounting for temporal correlation using adaptive repeatedmeasures methods. ${ }^{16}$ Two possible correlation structures are considered: the standard repeated-measures approach with constant correlations for different times and the more common longitudinal approach with order 1 spatial autoregressive correlations, which weaken the farther apart outcomes are in time. Variances are treated as constant over time as in standard repeated-measures analyses. 


\section{Results}

\section{Baseline characteristics of MINT study patients}

A total of 190 patients (equally randomized to each group) participated in the MINT study (Table 1). The majority of the patients were women (88.4\%), were $40-69$ years old $(77.9 \%)$, had a high school degree or less $(67.9 \%)$, were unmarried $(82.6 \%)$, were unemployed $(77.9 \%)$, had a reported annual income below $\$ 20,000$ (64.2\%), were on Medicaid (73.7\%), had uncontrolled baseline blood pressure (68.9\%), and/or had high baseline adherence self-efficacy of at least 3.5 out of $4(55.3 \%)$.

MEMS data were available for $141(74.2 \%)$ of the participants, with 70 (49.6\%) from the MINT group (Figure 1). Missing MEMS data was a consequence of a variety of factors, including lost or malfunctioning caps as well as patients not returning their MEMS caps for downloading at follow-up assessments. Patients with and without MEMS data did not differ significantly on any of the baseline characteristics (using $\chi^{2}$ or Fisher's exact test as appropriate). Hence, results for patients with MEMS data can be reasonably considered to be representative of results for the total sample.

\section{Individual-patient adherence patterns}

Observed adherence rates were modeled for each of the 141 patients with available MEMS data in terms of mean adherence and adherence variability over the 12-month study period. Results are plotted in Figure 2. These plots are similar to the chronology plots of Vrijens et $\mathrm{al}^{7}$ in depicting observed adherence rates (plotted as circles) over time and their variability, but they also provide fitted mean adherence curves (the middle curve of the plots) and adherence variability curves (the outer two curves of the plots) not addressed in chronology plots.

For patient 1, mean adherence was very high, at almost exactly the prescribed rate of one dose per day throughout the patient's participation period, and adherence variability was reasonably low although somewhat higher early in study participation. The observed adherence was $90.9 \%$ consistent with adherence at the prescribed rate. Patient 2 had high mean adherence, below but not too much below the prescribed rate and moderate adherence variability, being $48.1 \%$ consistent with adherence at the prescribed rate. Patient 3 had a convex mean adherence pattern, deteriorating somewhat early on but then improving to high by the end of study participation along with moderate to low adherence variability, for a percent consistency of $15.2 \%$.
Table I Baseline characteristics of patients in the MINT study

\begin{tabular}{|c|c|c|}
\hline Characteristic & $n$ & $\%$ \\
\hline \multicolumn{3}{|l|}{ Treatment group } \\
\hline Usual care & 95 & 50 \\
\hline MINT & 95 & 50 \\
\hline \multicolumn{3}{|l|}{ Gender } \\
\hline Female & 168 & 88.4 \\
\hline Male & 22 & 11.6 \\
\hline \multicolumn{3}{|l|}{ Age } \\
\hline$<40$ & 22 & 11.6 \\
\hline $40-69$ & 148 & 77.9 \\
\hline$\geq 70$ & 20 & 10.5 \\
\hline \multicolumn{3}{|l|}{ Education } \\
\hline Elementary school & 44 & 23.2 \\
\hline High school & 85 & 44.7 \\
\hline Some college or better & 61 & 32.1 \\
\hline \multicolumn{3}{|l|}{ Marital status } \\
\hline Single & 84 & 44.2 \\
\hline Married & 33 & 17.4 \\
\hline Separated or divorced & 58 & 30.5 \\
\hline Widowed & 15 & 7.9 \\
\hline \multicolumn{3}{|l|}{ Employment status } \\
\hline Full time & 29 & 15.3 \\
\hline Part time & 13 & 6.8 \\
\hline Retired & 20 & 10.5 \\
\hline Not working & 103 & 54.2 \\
\hline On disability & 25 & 13.2 \\
\hline \multicolumn{3}{|l|}{ Annual income level } \\
\hline Under $\$ 20,000$ & 122 & 64.2 \\
\hline$\$ 20,000$ or more & 39 & 15.3 \\
\hline Unknown & 29 & 20.5 \\
\hline \multicolumn{3}{|l|}{ Insurance status } \\
\hline Insurance plan or $\mathrm{HMO}$ & 13 & 6.8 \\
\hline Medicare & 22 & 11.6 \\
\hline Medicaid & 140 & 73.7 \\
\hline Self insurance & 15 & 7.9 \\
\hline \multicolumn{3}{|l|}{ Baseline SBP } \\
\hline Controlled (SBP < I40) & 80 & 42.1 \\
\hline Uncontrolled (SBP $\geq 140)$ & 110 & 57.9 \\
\hline \multicolumn{3}{|l|}{ Baseline DBP } \\
\hline Controlled (DBP < 90) & 94 & 52.1 \\
\hline Uncontrolled (DBP $\geq 90)$ & 91 & 47.9 \\
\hline \multicolumn{3}{|l|}{ Baseline BP } \\
\hline Controlled $(\mathrm{SBP}<130$ and $\mathrm{DBP}<90)$ & 59 & 31.1 \\
\hline Uncontrolled (SBP $\geq 140$ or $\mathrm{DBP} \geq 90$ ) & $|3|$ & 68.9 \\
\hline \multicolumn{3}{|l|}{ Baseline adherence self-efficacy } \\
\hline$<3.5$ & 85 & 44.7 \\
\hline$\geq 3.5$ & 105 & 55.3 \\
\hline \multicolumn{3}{|l|}{ MEMS data } \\
\hline None available & 49 & 25.8 \\
\hline Some available & $|4|$ & 74.2 \\
\hline
\end{tabular}

Note: Out of 190 African-American patients.

Abbreviations: BP, blood pressure; DBP, diastolic BP; SBP, systolic BP; HMO, Health Maintenance Organization; MEMS, Medication Event Monitoring System; MINT, motivational interviewing. 


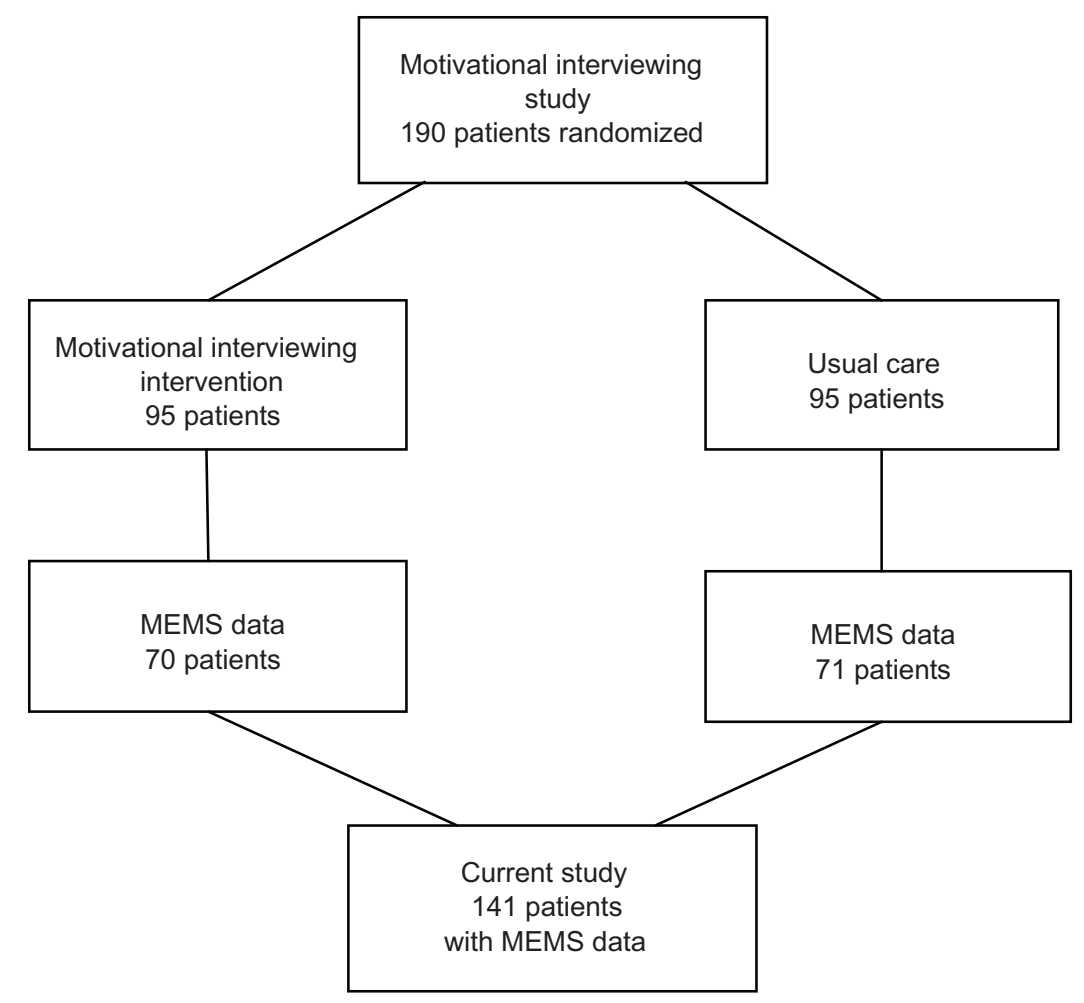

Figure I Patients from the parent motivational interviewing study and the current study.

Patients 1, 2, and 3 had either very high, high, or improving and thus acceptable adherence patterns, respectively. Other patients had deteriorating or very low and thus unacceptable adherence patterns. Patient 4 had mean adherence that deteriorated from very high to low over time, along with high to moderate adherence variability, for a percent consistency of $0.1 \%$. Patient 5 had mean adherence that gradually deteriorated from moderate to low over time and adherence variability that decreased from moderate to low, for a percent consistency of $0.1 \%$. Patient 6 had mean adherence that deteriorated from high to zero early on and adherence variability that decreased from low to zero, for a percent consistency of $0.1 \%$. Patient 7 had very low mean adherence over time and also very low adherence variability, for a percent consistency of $0.1 \%$.

\section{Categorization of adherence patterns into types}

Patients with MEMS data were clustered into groups based on their individual adherence patterns. The clusters were based on estimated mean adherence and adherence variability, computed at $5 \%, 10 \%, \ldots$, and $95 \%$ of time during study participation, for each of the 141 patients. For example, the study participation by patient 1 was 267 days, and so mean adherence and adherence variability estimates for this patient were computed at 13.4 (5\% of 267) days, 26.7 (10\% of 267$)$ days, ..., and 253.7 (95\% of 267) days.

Likelihood cross-validation scores were computed for multivariate normal mixture models, ${ }^{21}$ with means and variances treated as different across clusters, and with a common unstructured correlation matrix for all clusters to limit the number of parameters. Clusters were restricted to include at least $5 \%$ of the patients to avoid sparse clusters. The clustering approach with the best likelihood cross-validation score generated seven clusters using Ward's method based on standard Euclidean distance. ${ }^{22}$ Adherence types corresponding to these clusters are described in Table 2. Patients 1 to 7 were chosen as representative of clusters 1 to 7 . Averages of mean adherence and of adherence variability for patients in each cluster, as plotted in Figure 3, were used to determine Table 2 adherence type descriptions. These were similar to, but not always exactly the same as, mean adherence and adherence variability for patients 1 to 7 .

Cluster 1 corresponded to very high adherence, cluster 2 to high adherence, and cluster 3 to improving adherence. Thus, these three clusters represented acceptable adherence (ie, relatively high or improving). Clusters 4 to 6 corresponded to deteriorating adherence, while Cluster 7 corresponded to very low adherence. Thus, clusters 4 to 7 represented unacceptable adherence. These categorizations 
Patient $1-90.9 \%$ consistency

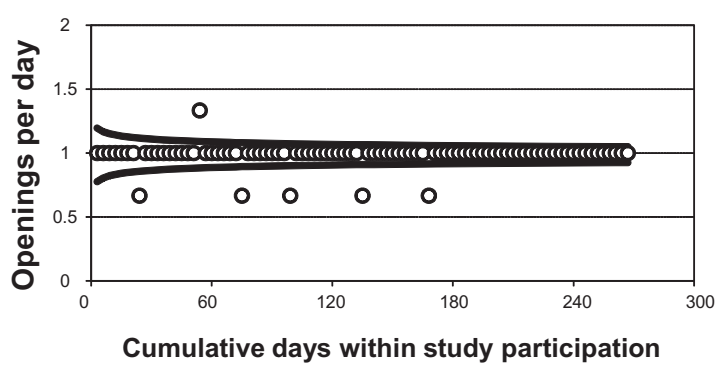

Patient $3-15.2 \%$ consistency

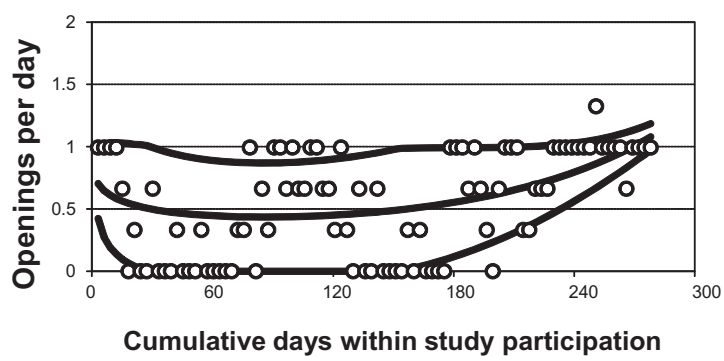

Patient $5-0.1 \%$ consistency

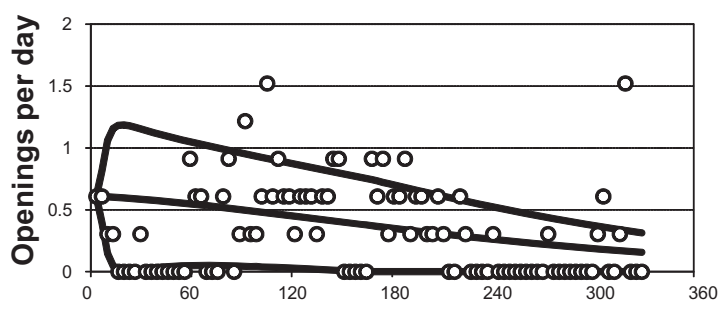

Cumulative days within study participation
Patient $2-48.1 \%$ consistency

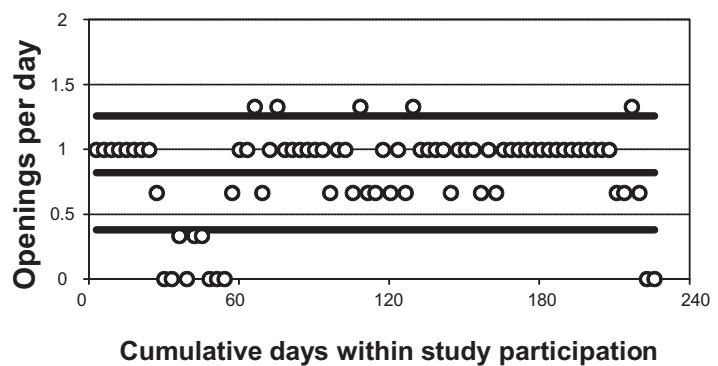

Patient $4-0.1 \%$ consistency

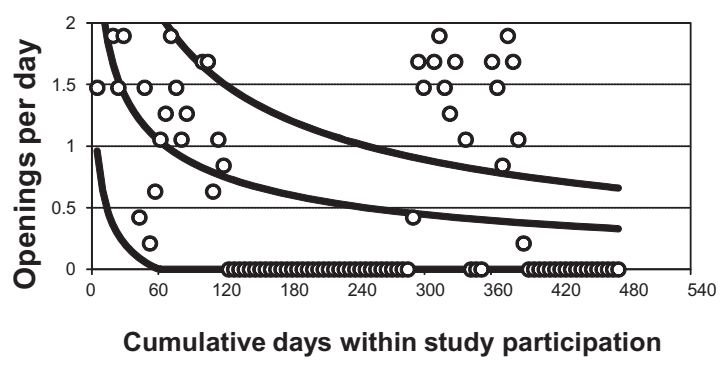

Patient $6-0.1 \%$ consistency

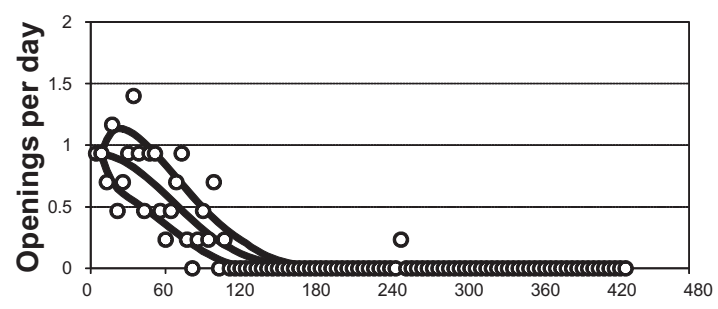

Cumulative days within study participation

Patient $7-0.1 \%$ consistency

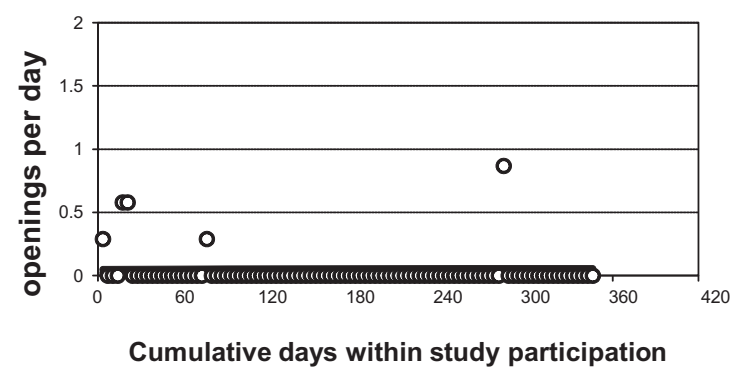

Figure 2 Example individual-patient adherence patterns.

of adherence were supported by decreasing average percent consistency and percent prescribed doses taken with increasing cluster indexes (Table 2). Note that cluster 5 corresponded to what Vrijens et $\mathrm{al}^{7}$ refer to as poor quality of execution, while clusters 4,6 , and 7 represented what they describe as short persistence (ie, disengagement from the dosing regimen). Note also that adherence was measured over the whole study period, including during periods of nonpersistence.

Patients with acceptable adherence were significantly $\left[\left(\chi^{2}(1)=76.05 ; P<0.001\right]\right.$ more likely to have a high percentage of prescribed doses taken (defined as at least $80 \%$ prescribed doses taken as in the parent study). However, only $70.8 \%$ of the patients with acceptable adherence as 
Table 2 Adherence clusters

\begin{tabular}{|c|c|c|c|c|c|c|}
\hline \multirow[t]{2}{*}{ Cluster } & \multirow[t]{2}{*}{$n$} & \multirow[t]{2}{*}{$\%$} & \multicolumn{2}{|l|}{ Adherence types } & \multirow{2}{*}{$\begin{array}{l}\text { Average \% } \\
\text { consistency }\end{array}$} & \multirow{2}{*}{$\begin{array}{l}\text { Average \% prescribed } \\
\text { doses taken }\end{array}$} \\
\hline & & & Mean adherence & Adherence variability & & \\
\hline I & 19 & 13.5 & Very high & Low & 80.8 & 97.2 \\
\hline 2 & 37 & 26.2 & High & Moderate & 50.6 & 84.9 \\
\hline 3 & 9 & 6.4 & Improving & Low to moderate & 14.1 & 55.1 \\
\hline 4 & 10 & 7.1 & $\begin{array}{l}\text { Deteriorating from } \\
\text { very high }\end{array}$ & $\begin{array}{l}\text { Deteriorating from } \\
\text { very high }\end{array}$ & I.I & 51.0 \\
\hline 5 & 18 & 12.8 & $\begin{array}{l}\text { Deteriorating from } \\
\text { moderate gradually }\end{array}$ & $\begin{array}{l}\text { Deteriorating from } \\
\text { moderate gradually }\end{array}$ & 7.7 & 42.3 \\
\hline 6 & 26 & 18.4 & $\begin{array}{l}\text { Deteriorating from } \\
\text { moderate early on }\end{array}$ & $\begin{array}{l}\text { Deteriorating from } \\
\text { moderate early on }\end{array}$ & 0.1 & 14.5 \\
\hline 7 & 22 & 15.6 & Very low & Very low & 0.1 & 5.8 \\
\hline Total & $|4|$ & 100 & & & & \\
\hline
\end{tabular}

determined by ASM also had high percent prescribed doses taken. This suggests that the categorization into acceptable and unacceptable adherence based on ASM differs distinctly from the commonly used categorization based on percent prescribed doses taken scores, underscoring the potential problems with assuming constant adherence over time.

The important study outcome of having uncontrolled blood pressure (ie, either systolic blood pressure $\geq 140 \mathrm{mmHg}$ or diastolic blood pressure $\geq 90 \mathrm{mmHg}$ ) at 12 months did not depend on having a high percentage of prescribed doses taken $\geq 80 \%\left[\chi^{2}(1)=0.24 ; P=0.627\right]$. However, the chance of having uncontrolled blood pressure at 12 months was significantly higher for patients with deteriorating adherence than for other patients $\left[\chi^{2}(1)=7.59 ; P=0.006\right]$.

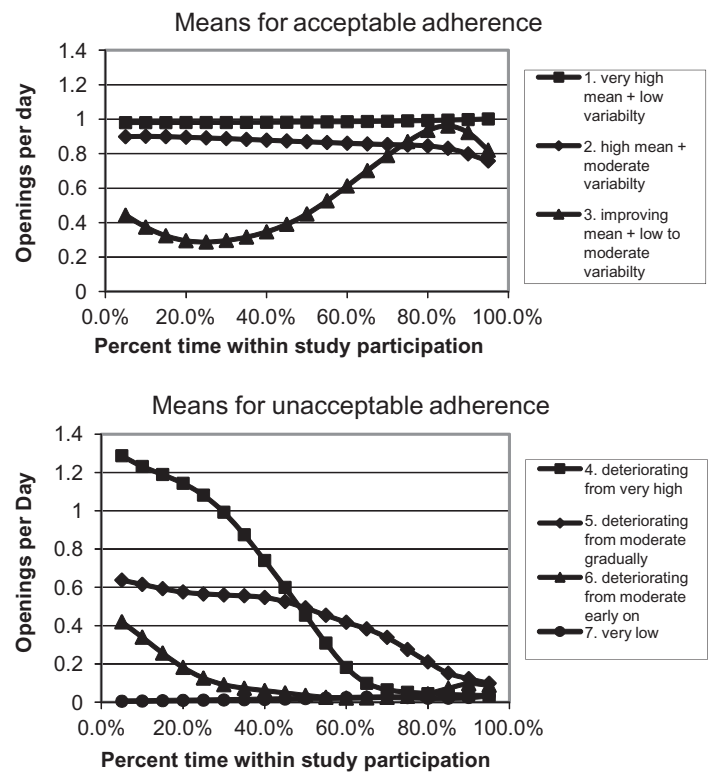

Figure 3 Average adherence patterns for adherence clusters.

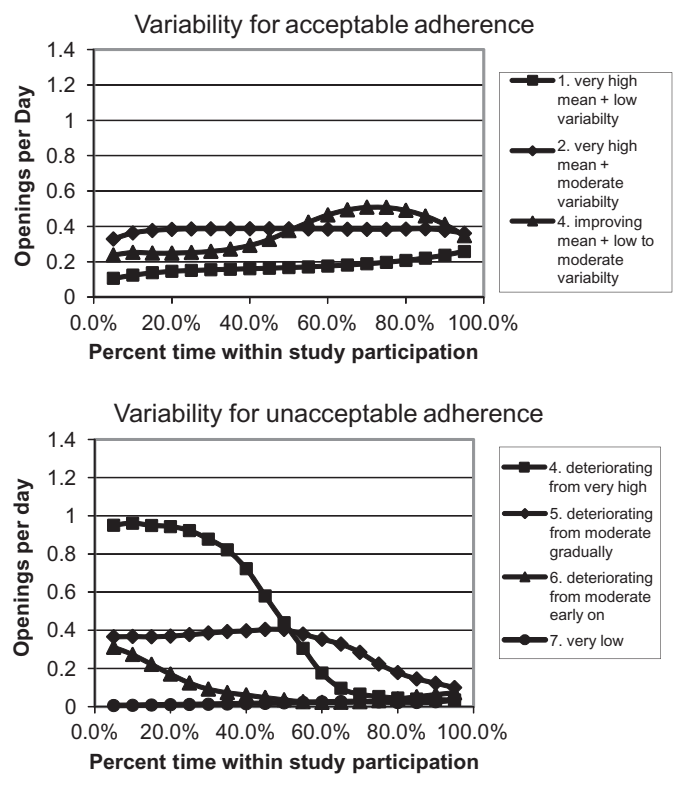

Adherence type effects on means for patient outcomes can be assessed using all seven adherence types or other categorizations of adherence, including very high versus not, either very high or high versus not, and acceptable (ie, very high, high, or improving) versus unacceptable. However, computing times can be quite long. For that reason, analyses of mean patient outcomes (adherence self-efficacy, systolic and diastolic blood pressure) were restricted to adherence categorized as acceptable or unacceptable.

\section{Adaptive analysis of adherence self-efficacy}

There were 687 adherence self-efficacy measurements for the 141 patients with available MEMS data over five possible time points, for an average of 4.9 measurements per patient. 
An adaptive analysis was conducted to determine the effects of adherence (acceptable versus unacceptable), treatment group (MINT versus usual care), time, and their interactions on mean adherence self-efficacy. Autoregressive correlations were used because these generated better likelihood crossvalidation scores than constant correlations.

The model generated for adherence self-efficacy depended on time, treatment group, and adherence type (Figure 4). There were two mean adherence self-efficacy patterns, one for patients in the MINT group with acceptable adherence and one for all other patients. Mean adherence self-efficacy started at the same baseline level and tended to increase over time, but post-baseline mean adherence self-efficacy was higher at all times for patients in the MINT group with more acceptable adherence than for other patients. These results suggest that there were MINT group improvements in mean adherence self-efficacy, but only for MINT patients with acceptable adherence, not for all patients in the MINT group. The estimated standard deviation for adherence self-efficacy was 0.41 , while estimated correlations decreased from 0.57 at 3 months apart to 0.11 at 12 months apart.

\section{Adaptive analyses of systolic blood pressure}

There were 568 systolic blood pressure measurements for 141 patients with available MEMS data over five possible time points, for an average of 4.0 measurements per patient. An ASM model was first generated for systolic blood pressure over these five time points. The results indicated that mean systolic blood pressure did not change significantly according to treatment group (MINT versus usual care) or adherence type (acceptable versus unacceptable). However, this could have been a consequence of not adjusting post-baseline systolic blood pressure values for baseline values, and so ASM analyses were also conducted to address this issue.

There were 427 post-baseline systolic blood pressure measurements for 137 patients with available MEMS data (four patients had only baseline systolic blood pressure measurements) over four possible post-baseline time points, for an average of 3.3 measurements per patient. ASM analyses used constant correlations because these generated better likelihood cross-validation scores than autoregressive correlations.

The ASM-generated model was based on baseline systolic blood pressure, time, and treatment group, but not on adherence type (Figure 5). For patients with low baseline systolic blood pressure, mean systolic blood pressure was essentially the same for both treatment groups and at all times. For patients with high baseline systolic blood pressure, mean post-baseline systolic blood pressure was lower for participants in the MINT group than those in the usual care group, with larger differences for increased baseline systolic blood pressures and at later times. The estimated constant standard deviation for post-baseline systolic blood pressure was 15.6. The estimated constant correlation was 0.29 .

\section{Adaptive analyses of diastolic blood pressure}

There were 568 diastolic blood pressure measurements for 141 patients with available MEMS data over five possible time points, for an average of 4.0 measurements per patient. As for diastolic blood pressure, analyses over all five time points identified no treatment group or adherence type effects, and thus post-baseline diastolic blood pressure was

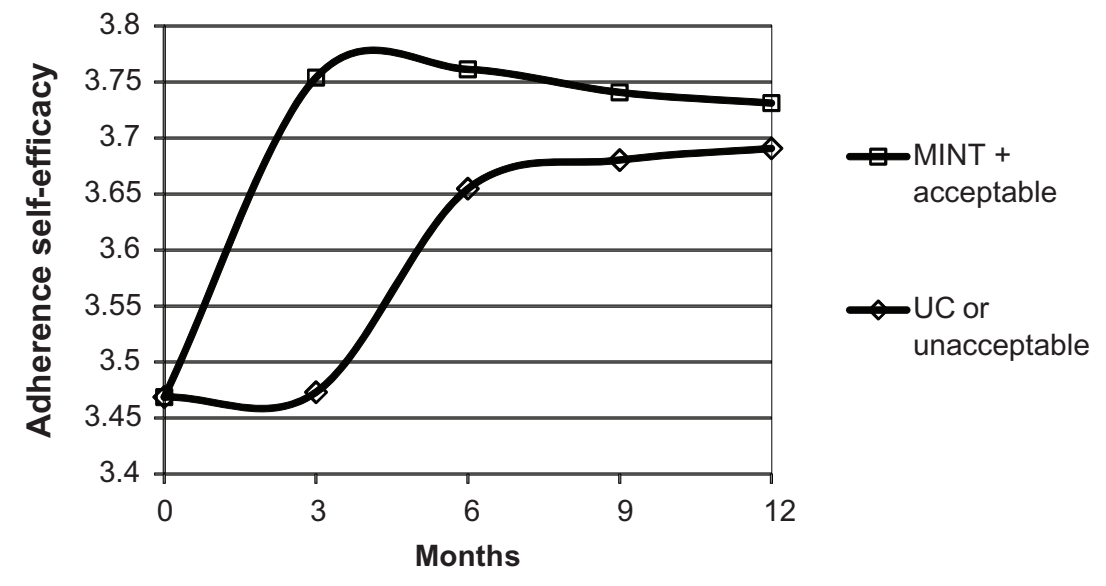

Figure 4 Mean adherence self-efficacy over time and by combinations of adherence type and treatment group. Abbreviations: MINT, motivational interviewing; UC, usual care. 


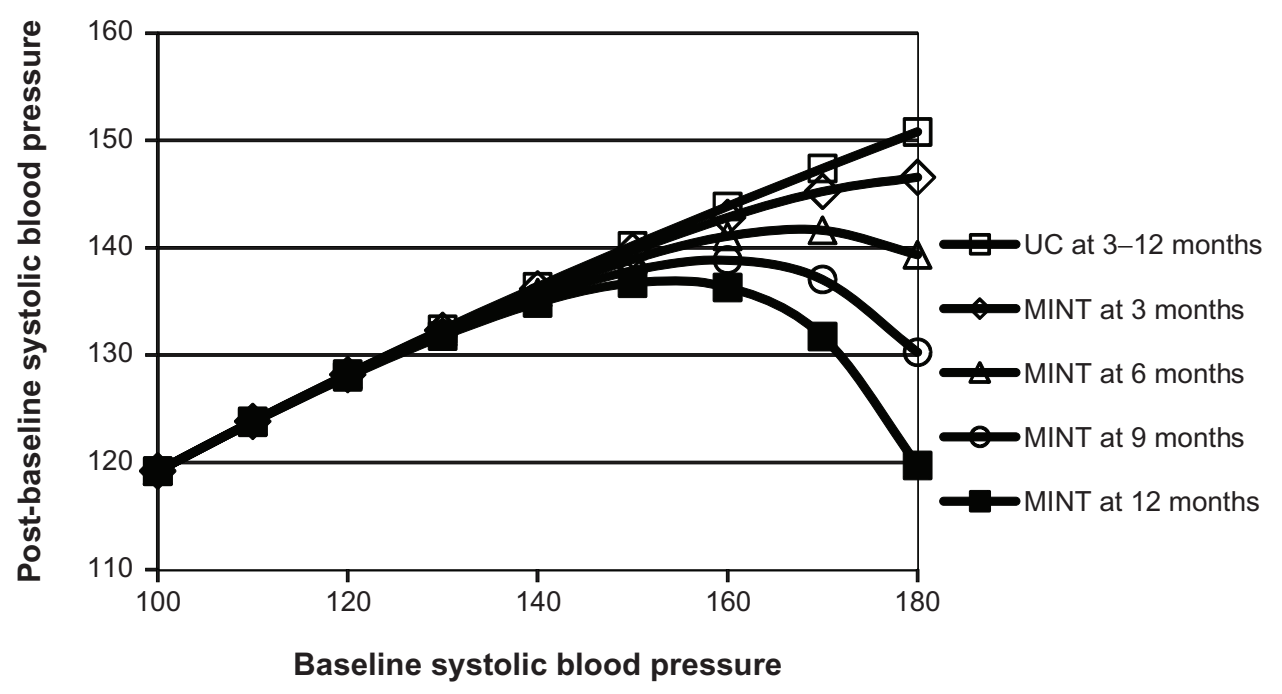

Figure 5 Mean post-baseline systolic blood pressure versus baseline systolic blood pressure over time and by treatment group. Abbreviations: MINT, motivational interviewing; UC, usual care.

analyzed controlling for baseline values. There were 427 post-baseline diastolic blood pressure measurements for 137 patients with available MEMS data (four patients had only baseline systolic blood pressure measurements) over the four possible post-baseline time points, for an average of 3.3 measurements per patient. Post-baseline diastolic blood pressure analyses used constant correlations because these generated better likelihood cross-validation scores than autoregressive correlations.

The ASM-generated model included time, treatment group, and adherence type effects (Figure 6). For patients with high baseline diastolic blood pressure values, mean post-baseline diastolic blood pressure was lower for participants in the MINT group than in the usual care group; the values decreased over time for patients in the MINT group and more so for those with very high baseline diastolic blood pressure when they had acceptable adherence than if they had unacceptable adherence. The estimated constant standard deviation for post-baseline diastolic blood pressure was 9.8. The estimated constant correlation was 0.23 .

\section{Comparison with standard repeated- measures modeling}

ASM results for mean adherence self-efficacy over all five time points were compared with results for the full factorial repeated-measures analysis of variance model in time, treatment group, adherence type, and all possible interactions. ASM results for mean systolic/diastolic blood pressure over the four post-baseline time points were compared with results for the full factorial repeated-measures analysis of covariance model in time, treatment group, adherence type, and all possible interactions, controlling for baseline systolic/diastolic blood pressure. All models assumed constant correlations and variances as is standard for repeated-measures modeling.

The model for mean adherence self-efficacy had three significant terms, ie, time $[F(4,530)=16.29 ; P<0.001]$, interaction of time with treatment group $[F(4,530)=4.64$; $P=0.001]$, and the interaction of time with adherence type $[F(4,530)=3.18 ; P=0.014]$. The model for post-baseline systolic blood pressure had only one significant term, ie, baseline systolic blood pressure $[F(1,132)=30.73 ; P<0.001]$. The model for mean diastolic blood pressure also had only one significant term, ie, baseline diastolic blood pressure $[F(1,132)=34.44 ; P<0.001]$.

\section{Discussion}

The goal of this paper was to elucidate the usefulness of ASM methods for providing novel insights into patterns of medication-taking and a better understanding of how that behavior affects patient outcomes. Analyses of data from a completed randomized controlled trial demonstrated that ASM methods revealed new information about individual patient adherence behaviors as well as about the dependence of changes in study outcomes over time on patient group assignment ie, MINT versus usual care. More importantly, the adherence behaviors that were identified using ASM methods in this paper were not apparent from the original analyses based on the conventional summary measure percent prescribed doses taken. ${ }^{14}$ Specifically, ASM methods were able to characterize patients as having several distinct adherence 

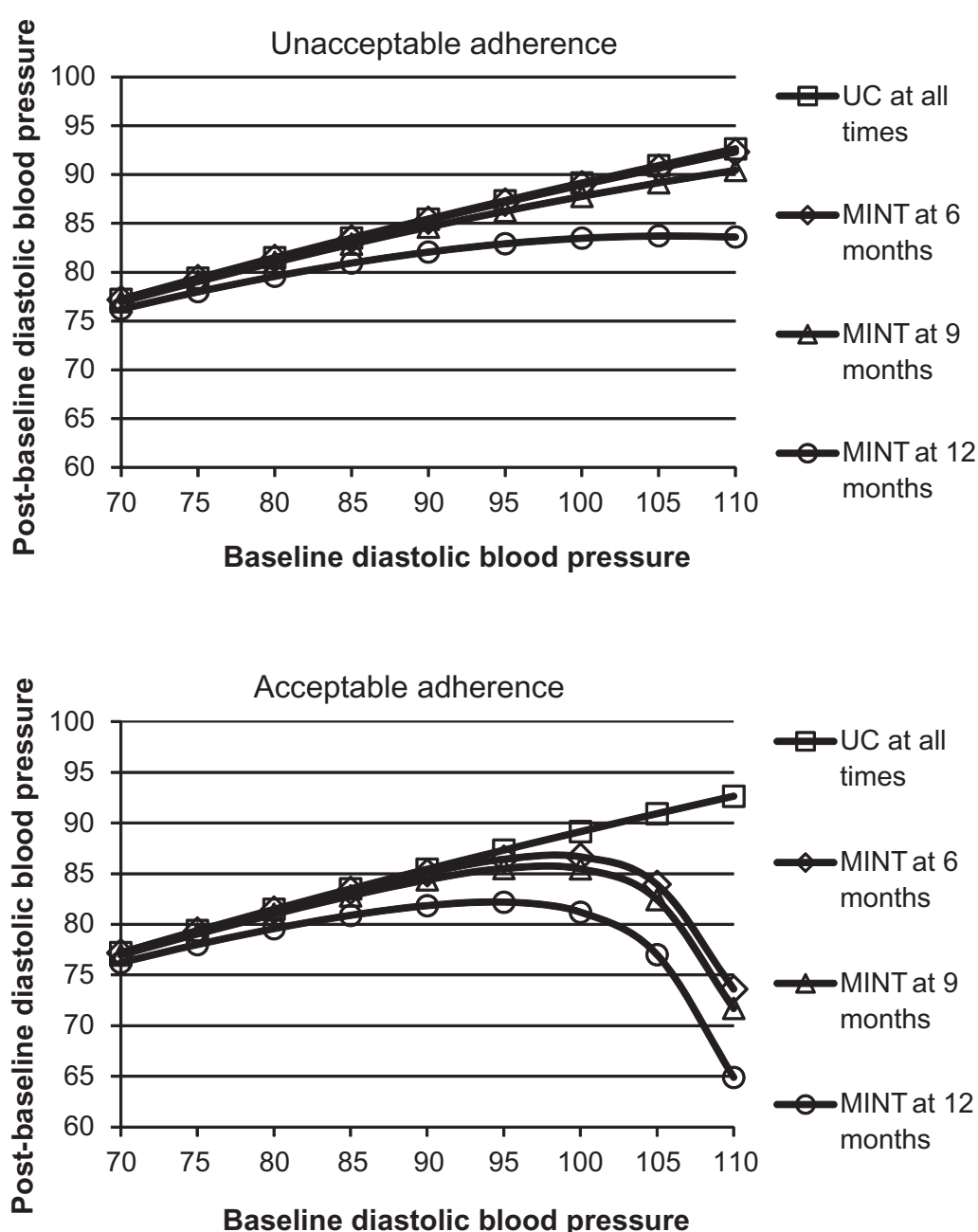

Figure 6 Mean post-baseline diastolic blood pressure versus baseline diastolic blood pressure over time and by combinations of adherence type and treatment group. Abbreviations: MINT, motivational interviewing; UC, usual care.

behaviors (very high, high, improving, deteriorating, or very low adherence) that were in contrast with the usual dichotomy of high adherence with percent prescribed doses taken represented as an adherence level of $\geq 80 \%$ or not. Very high, high, and improving adherence could be combined to produce the dichotomy of acceptable versus unacceptable adherence, but this was much different from high percent prescribed doses taken or not (ie, only $70.8 \%$ of patients with acceptable adherence also had high percent prescribed doses taken). The significance of the divergent findings that results from these contrasting methods is best exemplified by analysis of the blood pressure data from this trial, including systolic and diastolic blood pressure. Using conventional summary methods, high percent prescribed doses taken had no effect on blood pressure control at 12 months. In contrast, patients with deteriorating adherence, as determined through ASM methods, were more likely to have uncontrolled blood pressure at 12 months.
Furthermore, the results suggested that the intervention was most beneficial to patients with higher baseline blood pressure levels and acceptable adherence rather than for all patients who were randomized to the MINT group. Moreover, as expected, the MINT intervention had a positive influence on patient medication adherence self-efficacy. However, the effect was limited to those patients who also had acceptable adherence levels. In this case, the strength of the intervention appeared to lie in reinforcing patient confidence to maintain good adherence behaviors and might not have been suitable for addressing complex issues facing patients with poor adherence. Also, while the intervention targeted adherence behaviors, the effect of MINT on systolic blood pressure by the end of the study (12 months) was unrelated to adherence type. This finding attests to the multifactorial nature of blood pressure control. Types of medications and their properties and/or changes in lifestyle behaviors (ie, diet and exercise) were not measured in this 
trial, but might explain the observed reduction in systolic blood pressure.

Standard repeated-measures analysis of variance/ covariance models in time, treatment group, and adherence type with possible covariates can be overly complicated and so suggest that there are no treatment group or adherence type effects when in fact there are such effects (for example, this held for reported systolic and diastolic blood pressure analyses). ASM analyses are needed to identify which effects, if any, are of substance and which are not. Even when standard repeated-measures analyses identify significant effects (as for reported adherence self-efficacy analyses), ASM modeling provides a fuller depiction of those effects. The reported repeated-measures analyses demonstrate that ASM methods can provide parsimonious descriptions of how outcomes change with predictors like time, baseline values, treatment group, adherence type, and their interactions, and so identify distinct effects that standard repeated-measures models can sometimes suggest do not exist. While ASM methods were used here to analyze data from a study of electronically monitored adherence, they can also be used to perform adaptive analyses of repeated-measures data from studies addressing areas other than adherence.

Seven adherence types were identified in the analyses within three categories of relatively consistent adherence over time, deteriorating adherence over time, and improving adherence over time. The number of adherence types is likely to vary with the study under analysis, but variations on these three categories of adherence types can be expected to be identified for any type of medication. For example, Knafl et $\mathrm{al}^{13}$ identified 10 adherence types for subjects with human immunodeficiency virus (HIV) on antiretroviral medications, including seven relatively consistent, one deteriorating, and two improving adherence types. However, these adherence patterns were also identified in the context of a randomized controlled trial, so it is not clear if the same adherence patterns would emerge in clinical settings.

In summary, using ASM methods, this study identified specific patient-related and disease-related factors (for example, treatment group, adherence type, and baseline systolic/ diastolic blood pressure) that jointly predicted improvements in psychosocial outcomes (ie, adherence self-efficacy) and in clinical outcomes (ie, post-baseline systolic/diastolic blood pressure) in a sample of African-American patients enrolled in a motivational interviewing trial. In some cases, these effects were not identifiable with standard repeatedmeasures methods currently used for such assessments and their distinct nonlinearity in none of the cases.

\section{Limitations}

The adherence data analyzed in this article were collected electronically using MEMS caps. Cap openings do not always necessarily correspond to actual medication-taking. Patients may have sometimes removed multiple doses at one cap opening in order to put them in pill boxes, in which case the cap openings underestimate actual adherence. On the other hand, patients were enrolled in a randomized controlled trial addressing adherence and so they may have sometimes opened the cap to appear adherent without actually taking any medications, in which case the cap openings overestimate actual adherence. However, positive effects on adherence self-efficacy and blood pressure identified in the analyses for acceptable compared with unacceptable adherence, as determined by MEMS cap openings, suggest that, for most patients in the MINT study, their MEMS cap data reflect their actual adherence quite closely.

The reported findings provide useful information for use in the research field of adherence to medication regimens. However, beneficial effects of improvements in patient medication-taking behaviors on blood pressure control and cardiovascular events remain to be determined in future investigations.

There are also limitations to current ASM methods that should be noted and these require further research. First, while only adaptive Poisson regression of counts/rates is needed for modeling EMD adherence data, ASM methods currently also support adaptive linear and logistic regression modeling of uncorrelated continuous and discrete outcomes, respectively. However, ASM methods currently only support adaptive repeated-measures modeling of correlated continuous outcomes. There is a need to extend these to support adaptive modeling of correlated counts/rates and correlated discrete outcomes. Second, ASM methods currently support only two correlation structures for repeated-measures analyses, which are constant as in standard repeated-measures modeling and order 1 autoregression. Extensions are needed to handle more general correlation structures, for example, random-effect models. Third, other methods could have been used. For example, latent class analysis could be used instead of standard clustering procedures. Delucchi et al ${ }^{12}$ considered both these approaches for determining adherence types for HIV-positive methadone patients and found that there was good agreement between the two alternative cluster assignments, but adaptive analyses were still needed to generate the adherence patterns they clustered. Fourth, in this study, variability was modeled only for adherence while standard constant variance models were used for analyzing 
longitudinal outcomes. Further research is needed to address the impact of heterogeneous variance on the conclusions for repeated-measures analyses. Fifth, the analyses only addressed the frequency of medication-taking, not the timing of medication-taking. Sixth, ASM analyses can require extensive computation time. Finally, ASM methods currently are not directly supported by statistical software tools. However, they have been implemented in SAS macros available from the corresponding author.

\section{Conclusion}

Over the past 30 years, numerous interventions have been developed to improve medication adherence in hypertensive patients; however, no single strategy has emerged as superior for all patients. ${ }^{23}$ While complex intervention strategies have proven more successful than simple strategies, ${ }^{24}$ they too have failed to demonstrate sizable effects that justify the high costs and resources needed for implementation. ${ }^{25}$ Rather than developing more complex, resource-intensive interventions, new innovative methods are needed that directly address the multifaceted nature of patient medication-taking behaviors. ASM methods provide an approach for developing tailored and potentially cost-effective intervention strategies to improve adherence in patients who are at greatest risk for poor adherence, such as those with lower socioeconomic status and who are in racial/ethnic minorities. ${ }^{26}$

The findings of this study have several important implications that can guide future intervention efforts. First, examining individual adherence patterns can assist in developing patient-specific intervention strategies that could maximize efforts to improve patient's medication-taking behaviors. For example, patients who are nonadherent due to self-termination of their medication require counseling approaches that build confidence and motivation to continue with treatment, even in situations that may pose challenging. Alternatively, patients whose nonadherence is due to problems with execution of the regimen require counseling approaches that assist in developing behavioral action plans and identifying sources of social support to assist in taking medications as prescribed.

ASM methods could also be applied to future clinical trials by the inclusion of a run-in phase during the first month of the trial to generate adherence types and stratify patients according to their adherence status as well as other key psychosocial and clinical characteristics. This initial groundwork could help researchers to determine which intervention approach would be best matched to individual patient needs and to facilitate medication adherence behaviors. It is crucial that this preliminary assessment be made close to randomization into treatment groups because it is not clear whether a patient's long-term history of medication taking is as good an indicator of that patient's present or future medication-taking behavior. For example, patients may have been consistently adherent until a future significant life event/stressor occurs and interferes with medication-taking behavior, after which their adherence deteriorates. These kinds of events may be the root cause for some deteriorating individual patient adherence patterns. Research is needed on what length of a run-in period would be most effective for predicting subsequent medication-taking behavior.

\section{Acknowledgment}

This work was supported in part by grants R01 HL69408, K23 HL098564, and K24 HL111315 from the National Heart, Lung, and Blood Institute and grant P60 MD003421 from the National Center for Minority Health Disparities of the National Institutes of Health. The content is solely the responsibility of the authors and does not necessarily represent the official views of the National Heart, Lung, and Blood Institute, the National Center for Minority Health Disparities, or the National Institutes of Health.

\section{Disclosure}

The authors report no conflicts of interest in this work.

\section{References}

1. Brunenberg DE, Wetzels GE, Nelemans PJ, et al. Cost effectiveness of an adherence-improving programme in hypertensive patients. Pharmacoeconomics. 2007;25:239-351.

2. Düsing R, Handrock R, Klebs S, Tousset E, Vrijens B. Impact of supportive measures on drug adherence in patients with essential hypertension treated with valsartan: the randomized, open-label, parallel group study VALIDATE. J Hypertens. 2009;27:894-901.

3. Gerin W, Tobin JN, Schwartz JE, et al. The medication Adherence and Blood Pressure Control (ABC) trial: a multi-site randomized controlled trial in a hypertensive, multi-cultural, economically disadvantaged population. Contemp Clin Trials. 2007;28:459-471.

4. Ogedegbe G, Schoenthaler A, Richardson T, et al. An RCT of the effect of motivational interviewing on medication adherence in hypertensive African Americans: rationale and design. Contemp Clin Trials. 2007;28:169-181.

5. Rose AJ, Berlowitz DR, Manze M, Orner MB, Kressin NR. Intensifying therapy for hypertension despite suboptimal adherence. Hypertension. 2009;54:524-529.

6. Schoenthaler A, Ogedegbe G. Patients' perceptions of electronic monitoring devices affect medication adherence in hypertensive African Americans. Ann Pharmacother. 2008;42:647-652.

7. Vrijens B, Vincze G, Kristanto P, Urquhart J, Burnier M. Adherence to prescribed antihypertensive drug treatments: longitudinal study of electronically compiled dosing histories. BMJ. 2008;336:1114-1117.

8. Sereika SM, Dunbar-Jacob J. Analysis of electronic event monitored adherence. In: Burke LE, Ockene IS, editors. Compliance in Healthcare and Research. Armonk, NY: Futura Publishing; 2003.

9. Knafl GJ, Fennie KP, Bova C, Dieckhaus K, Williams AB. Electronic monitoring device event modelling on an individual-subject basis using adaptive Poisson regression. Stat Med. 2004;23:783-801. 
10. Aloia MS, Goodwin MS, Velicer WF, et al. Time series analysis of treatment adherence patterns in individuals with obstructive sleep apnea. Ann Behav Med. 2008;36:44-53.

11. Knafl GJ, Bova CA, Fennie KP, O’Malley JP, Dieckhaus KD, Williams AB. An analysis of electronically monitored adherence to antiretroviral medications. AIDS Behav. 2010;14:755-768.

12. Delucchi KL, Knafl GJ, Haug N, Sorensen J. Adaptive Poisson modeling of medication adherence among HIV-positive methadone patients provided greater understanding of behavior. In: Ekwall B, Cronquist M, editors. Micro Electro Mechanical Systems (MEMS) Technology, Fabrication Processes and Applications. Hauppauge, NY: Nova Science Publishers; 2010. Available from: https://www.novapublishers. com/catalog/product_info.php?products_id=19148. Accessed February 29, 2012.

13. Knafl GJ, Delucchi KL, Bova CA, Fennie KP, Ding K, Williams AB. A systematic approach for analyzing electronically monitored adherence data. In: Ekwall B, Cronquist M, editors. Micro Electro Mechanical Systems (MEMS) Technology, Fabrication Processes and Applications. Hauppauge, NY: Nova Science Publishers; 2010. Available from: https://www.novapublishers.com/catalog/product_info.php?products_ id=19133. Accessed February 29, 2012.

14. Ogedegbe GO, Chaplin W, Schoenthaler AS, et al. A practice-based trial of motivational interviewing and adherence in hypertensive African Americans. Am J Hypertens. 2008;21:1137-1143.

15. Ogedegbe O, Mancuso CA, Allegrante JP, Charlson ME. Development and evaluation of a medication adherence self-efficacy scale in AfricanAmerican patients. J Clin Epidemiol. 2003;56:520-529.

16. Knafl GJ, Fennie KP, O’Malley JP. Adaptive repeated measures modeling using likelihood cross-validation. In: Bovaruchuk B, editor. Proceedings Second IASTED International Conference on Computational Intelligence. Anaheim, CA: ACTA Press; 2006.
17. Royston P, Altman DG. Regression using fractional polynomials of continuous covariates: parsimonious parametric modelling. Appl Stat. 1994;43:429-467.

18. McCullagh P, Nelder JA. Generalized Linear Models. Boca Raton, FL: Chapman \& Hall/CRC; 1999.

19. Knafl GJ, Grey M. Factor analysis model evaluation through likelihood cross-validation. Stat Methods Med Res. 2007;16:77-102.

20. Stone M. An asymptotic equivalence of choice of model by crossvalidation and Akaike's criterion. J Roy Stat Soc B. 1977;39:44-47.

21. Symons MJ. Clustering criteria and multivariate normal mixtures. Biometrics. 1981;37:35-43.

22. SAS Institute Inc. SAS/STAT 9.1 User's Guide. Cary, NC: SAS Institute; 2004.

23. Takiya LN, Peterson AM, Finley RS. Meta-analysis of interventions for medication adherence to antihypertensives. Ann Pharmacother. 2004;38:617-624.

24. McDonald HP, Garg AX, Haynes RB. Interventions to enhance patient adherence to medication prescriptions: scientific review. JAMA. 2002;288:2868-2879.

25. Haynes RB, Ackloo E, Sahota N, McDonald HP, Yao X. Interventions for enhancing medication adherence. Cochrane Database Syst Rev. 2008;2:CD000011.

26. Hyre AD, Krousel-Wood MA, Muntner P, Kawasaki L, DeSalvo KB. Prevalence and predictors of poor antihypertensive medication adherence in an urban health clinic setting. J Clin Hypertens. 2007;9:179-186.
Patient Preference and Adherence

\section{Publish your work in this journal}

Patient Preference and Adherence is an international, peer-reviewed, open access journal focusing on the growing importance of patient preference and adherence throughout the therapeutic continuum. Patient satisfaction, acceptability, quality of life, compliance, persistence and their role in developing new therapeutic modalities and compounds to

\section{Dovepress}

optimize clinical outcomes for existing disease states are major areas of interest. This journal has been accepted for indexing on PubMed Central. The manuscript management system is completely online and includes a very quick and fair peer-review system. Visit http://www.dovepress.com/ testimonials.php to read real quotes from published authors. 